INVESTIGACIONES

de HISTORIA ECONÓMICA

2007, invierno, número 7. Pp. 69 a 100

\title{
La transición nutricional en la España contemporánea: las variaciones en el consumo de pan, patatas y legumbres (1850-2000)
}

\author{
The nutritional transition in contemporary Spain: \\ the evolution of the intake of bread, potatoes and pulses (1850-2000)
}

\author{
XAVIER CUSSÓ SEGURA \\ RAMÓN GARRABOU SEGURA \\ Universitat Autónoma de Barcelona
}

\begin{abstract}
RESUMEN
ABSTRACT

En este artículo se analiza la transición nutricional moderna en España. Un proceso que se inicia en el siglo XIX, que se traduce en una progresiva mejora de la alimentación y del estado nutritivo de la población hasta la década de 1930, que se interrumpe durante prácticamente dos décadas y que se reemprende de forma definitiva en la segunda mitad del siglo XX. El trabajo se centra en la evolución del consumo de tres grupos de alimentos que han representado una parte

fundamental de la dieta de la población española hasta el último tercio del siglo XX: los cereales, las patatas y las legumbres secas. Asimismo, se compara el proceso español con las transiciones nutricionales que experimentan otros países de Europa occidental, mostrando su moderado retraso y su convergencia final.

PALABRAS CLAVE: Transición nutricional, Sistema alimentario, Consumo de alimentos, España contemporánea

KEY WORDS: Nutritional transition, Food system, Food consumption, Contemporary Spain

Códigos JEL: N33, N34

This article analyses the contemporary nutritional changes of Spain. The process begins in the 19th century with the progressive improvement in food intake and in the nutritional status of the population up to 1930. This trend is interrupted for twenty years and begins to improve again definitively during the second half of the 20th century. This study focuses on the evolution of the intake of three central food groups of the Spanish diet up to the end of the 20th century: cereals, potatoes and pulses. This progression is also compared to the nutritional changes of other West European countries, highlighting its moderate delay and its final convergence.

JEL Codes: N33, N34
\end{abstract}




\section{Introducción ${ }^{1}$}

$\mathrm{U}$ na de las manifestaciones más destacadas del desarrollo económico moderno ha sido el cambio profundo que se ha producido en los sistemas alimentarios. Investigadores como Smil (2000) han propuesto el concepto de transición nutricional para identificar las modificaciones fundamentales y los factores que las han impulsado. Incrementos de la renta, crecimiento de la producción agraria y ganadera, progreso científico, formación de los mercados de productos agrarios, procesos de urbanización e incorporación de la mujer al trabajo, entre otros, han sido los principales motores de estos cambios.

La transición nutricional se inició en el siglo XIX simultáneamente al proceso de industrialización y, en una primera etapa, consistió básicamente en regularizar para el conjunto de la población una ingesta calórica suficiente. Las mejoras en la producción y distribución de alimentos, y el incremento de los ingresos reales se tradujeron en un mayor consumo de bienes de subsistencia, en particular cereales, tubérculos y legumbres; así como en un incremento moderado del consumo de productos como el café, el azúcar o el chocolate. En la etapa siguiente, a pesar del crecimiento de la renta, el consumo de alimentos básicos como los cereales o las patatas se estabilizó y pronto empezó a descender, a la vez que creció el consumo de alimentos de origen animal, hortalizas, frutas y azúcares. Al final del proceso, la dieta había cambiado profundamente. De unas ingestas calóricas inferiores a menudo a las 2.500 kilocalorías por habitante y día, propias de las sociedades preindustriales, se pasa, al final de la transición nutricional, a superar las 3.000, generando problemas de obesidad, que representan un nuevo y grave deterioro de la salud. De unas dietas monótonas en las que el pan, las patatas y leguminosas aportaban gran parte de la energía y proteínas, se pasa a un nuevo sistema alimentario en el que el consumo de carne, leche, grasas animales, azúcares, frutas y hortalizas tienen un peso muy importante, al mismo tiempo que se produce una notable reducción de cereales, patatas y legumbres. La práctica desaparición de las últimas de la dieta de los países ricos fue simultánea al crecimiento del consumo de carnes, huevos y productos lácteos, que apor-

[Fecha de recepción del original, octubre de 2005. Versión definitiva, junio de 2006]

Este trabajo se enmarca en el proyecto SEC2003-08-449, "Requerimientos y disponibilidades territoriales: cambios económicos en el uso del suelo y evolución histórica de los paisajes agrarios mediterráneos", financiado por el Ministerio de Ciencia y Tecnología. Versiones previas del mismo se han presentado en las Jornadas científicas “El bienestar y los niveles de vida en la España contemporánea" (Murcia, marzo de 2005), en el XI Congreso de la Sociedad Española de Historia Agraria (Aguilar de Campoo, junio de 2005) y en el VIII Congreso de la Asociación Española de Historia Económica (Santiago de Compostela, septiembre de 2005). Agradecemos a los organizadores, los ponentes y asistentes sus comentarios. Las sugerencias realizadas por los evaluadores anónimos y los editores de Investigaciones de Historia Económica también han contribuido a mejorar este trabajo. Las deficiencias sólo pueden imputarse a los autores. 
tan la mayor parte de las proteínas requeridas. Así se rompió uno de los elementos básicos de los sistemas alimentarios preindustriales: la función decisiva de la aportación de proteínas de las leguminosas como complemento de los cereales.

Hasta aquí los elementos caracterizadores de la transición alimenticia. El objetivo de nuestro trabajo es reconstruir las grandes etapas de su implantación en España. Nos centraremos en dos aspectos fundamentales: los cambios en la ingesta calórica entre mediados del siglo XIX y finales del XX, y, sobre todo, en las variaciones del consumo de cereales, tubérculos y legumbres en este período ${ }^{2}$. Como en otros aspectos de nuestra historia económica y social, existe una interpretación dominante, que destaca el considerable retraso de la transición nutricional en nuestro país en comparación con los países europeos de nuestro entorno.

La principal herramienta para estimar, con una perspectiva temporal y una amplitud geográfica aceptables y comparables, el consumo de nutrientes y de alimentos básicos en poblaciones del pasado son las hojas de balance de alimentos. Tienen generalmente ámbito estatal y están construidas a partir de las cifras elaboradas por la Administración y otras estimaciones sobre producciones, intercambios exteriores, pérdidas y usos diversos. Consisten en sumar, para cada alimento, la producción y las importaciones registradas en un período concreto, normalmente un año. De la cifra obtenida se resta la parte del producto destinada a semilla, a alimentación animal, a otros usos industriales, a exportaciones y, finalmente, las pérdidas. Se consideran también, en uno u otro sentido, las variaciones de existencias. El resultado se divide por la población y por el número de años comprendidos en el período estudiado, si éste es superior al año. En el caso español, sobre la producción se dispone de cifras de numerosos alimentos procedentes de estimaciones y cálculos realizados por los funcionarios y autoridades responsables, o por especialistas, que aparecen aisladamente o de forma más sistemática en publicaciones de la época, en los Anuarios de Estadística Agraria (AEA), o en compendios recientes de las cifras disponibles para el pasado, y que han sido en algunos casos recientemente corregidas en la medida que ha sido posible ${ }^{3}$. Disponemos de estimaciones sobre la parte destinada a semilla para diversos productos, y también de las cifras oficiales de exportaciones e importaciones recogidas en las estadísticas de comercio exterior, pero no conocemos con precisión, y en ciertos casos prácticamente desconocemos, las variaciones anuales de las existencias de cada alimento; las pérdidas que se producen en el almacenamiento, procesado, distribución y consumo; y la parte destinada a consumo ani-

Sin olvidar que las medias españolas ocultan grandes disparidades regionales en el consumo de los tres grupos de alimentos.

3 Véanse las cifras recogidas por la Junta Consultiva Agronómica (1904, 1905 y 1906) y por Sotilla (1981); la recopilación y análisis realizados por el Grupo de Estudios de Historia Rural (GEHR, en adelante) en diversos artículos y, especialmente, en GEHR (1991); o los recogidos por la FAO (Faostat). 
mal y a usos industriales, sobre todo a medida que nos remontamos en el tiempo. Tampoco tenemos suficiente información sobre las producciones para el autoconsumo en pequeños huertos, la disponibilidad e importancia de otros alimentos provenientes de la pequeña ganadería doméstica (aves, conejos, cerdos), la caza, la pesca marítima y fluvial, y la recolección en bosques. Por último, buena parte de la información disponible presenta algunos problemas que cabe también considerar. Por una parte, los factores de conversión de los alimentos en nutrientes se basan en la composición actual de estos alimentos, que puede diferir considerablemente, en ciertos casos, de la de otras épocas. Por otra, a partir de las hojas de balance trabajamos con consumos medios; la información sobre la distribución real de los alimentos y de los nutrientes entre la población es escasa, y la existente se refiere casi siempre a calorías y se basa en aproximaciones a través de las estimaciones que se hacen de la distribución de la renta, o también de los alimentos, en otros países o épocas ${ }^{4}$.

Para completar las informaciones sobre las ingestas de nutrientes procedentes de las hojas de balance también disponemos de encuestas de consumo, o en su defecto de presupuestos familiares (EPF), que no tienen una representatividad de carácter estatal hasta la década de 1960, pero que aportan datos interesantes y precisos para diversas épocas y grupos humanos, en especial, de forma más amplia, desde la década de $1950^{5}$. Por último, como complemento de la información descrita más arriba, tenemos las referencias cuantitativas y cualitativas procedentes de asignaciones, raciones, menús y presupuestos que aparecen en la documentación de asilos, hospitales, prisiones, cuarteles militares, escuelas, instituciones religiosas y anuarios; o se recogen en estudios científicos, como topografías médicas, investigaciones oficiales, descripciones de viajeros o testimonios diversos. Las encuestas de consumo y las otras fuentes cuantitativas y cualitativas sí que proporcionan datos sobre la distribución social y regional de los alimentos y nutrientes. Este tipo de información es relativamente abundante y de variada procedencia, aunque con escasa continuidad en el tiempo y limitada representatividad demográfica y geográfica, o sólo cubren períodos recientes. A pesar de los inconvenientes citados, consideramos que estas informaciones aportan aproximaciones bastantes fiables sobre el suministro de alimentos y las disponibilidades medias de energía y otros nutrientes por habitante, aunque se refieran a grupos sociales y a territorios muy concretos.

4 Para conocer la distribución real de la ingesta de vitaminas y minerales entre la población tendríamos que conocer la distribución detallada de los alimentos donde se encuentran cada uno de ellos, circunstancia que para el pasado resulta muy complicada.

5 Para el siglo XIX y la primera parte del XX, véanse los datos recogidos por la Comisión de Reformas Sociales y, posteriormente, por el Instituto de Reformas Sociales (Giral, 1914). Para la segunda mitad del XX, Varela, Moreiras-Varela y Vidal (1968); Varela, García Rodríguez y Moreiras-Varela (1971); o la explotación que el propio Varela y su equipo realizan de la Encuesta de Presupuestos Familiares de 1991 (Varela, 1995). 
El contraste de los datos disponibles -en algunos casos de carácter general, como las estimaciones de consumo aparente; en otros de carácter más particular, referidos a instituciones, estimaciones de algunos higienistas o bien encuestas de consumo familiar - con informaciones similares de otros países europeos, nos permitirán precisar la cronología de la transición nutricional en nuestro país y comprobar, a la vez, la existencia o no de retrasos significativos respecto a otros países europeos.

\section{El aumento y regularización de la ingesta calórica en la primera etapa de la transición nutricional}

La primera fase de la transición nutricional en los países europeos consistió en el incremento y regularización de una ingesta calórica que cubriera de forma suficiente los requerimientos nutricionales de la mayor parte de la población. Desde finales del siglo XIX se realizaron las primeras estimaciones sobre las necesidades energéticas, y estudios posteriores han establecido la cantidad de energía que se necesita para mantener las funciones vitales del cuerpo humano, el crecimiento y desarrollo del organismo, y las necesidades para el trabajo y actividades del ocio. Evidentemente, existen diferencias debidas a la edad, género y actividad, pero en 1950 la Organización de las Naciones Unidas para la Agricultura y la Alimentación (FAO) propuso unos valores de referencia, para un joven de 25 años y de $65 \mathrm{Kg}$. de peso y una mujer de la misma edad y $50 \mathrm{Kg}$. de peso, que consuman una dieta equilibrada, realicen actividades moderadas y vivan en climas templados. Las propuestas eran de 3.200 kilocalorías/día para el hombre y 2.300 para la mujer. En 1971 estos valores se modificaron ligeramente a la baja, fijándose en 3.000 y 2.200 kilocalorías/día, respectivamente. De nuevo, en 1985, se rectificaron a la baja: 2.978 y 2.018 kilocalorías/día para los mismos casos de referencia ${ }^{6}$. Asimismo, se asumió que en las sociedades preindustriales la ingesta calórica media se situaba por debajo de estos valores y que fue en el curso del siglo XIX cuando en los países industrializados europeos el consumo medio alcanzó aquellas cifras, y con ello un aceptable nivel de cobertura de sus necesidades. La mejora de la producción agraria y la estructuración de un mercado de bienes alimenticios básicos eliminaron las crisis de subsistencia, facilitando un abastecimiento regular, en particular para aquellos segmentos de la población con menos recursos. La demanda de bienes de subsistencia se mostró muy elástica al crecimiento de la renta,

6 Smil (2000), pp. 225-226. Para la población española en el siglo XX hemos estimado unos requerimientos energéticos medios por habitante entre 2.200 y 2.300 kilocalorías diarias (considerando su estructura por edades, sexos, actividad, etc.). Cussó (2005). 
en particular para las capas pobres de la población, que con anterioridad no conseguían cubrir de forma adecuada, a menudo, las necesidades mínimas de energía.

¿Cuál fue la cronología de la difusión de esta primera etapa de la transición nutricional? Aunque en algunos países europeos ya desde mediados del siglo XVIII se observan ciertas mejoras, los progresos significativos en la alimentación, y paralelamente en la esperanza de vida y en la salud, no se generalizaron hasta finales de la centuria siguiente. Los estudios disponibles para algunos países europeos indican que fue en la segunda mitad del siglo XIX cuando se produjeron avances decisivos, hasta llegar a finales de siglo a unos niveles de ingesta calórica media que podían cubrir de forma adecuada las necesidades energéticas de la mayoría de la población. Así lo muestra, por ejemplo, el caso de Francia, recogido en el Cuadro 1.

Aparte de evidenciar que a comienzos del siglo XX, en la mayoría de los países industrializados, se habían alcanzado unos niveles de ingesta calórica adecuados, el Cuadro 1 muestra que durante la centuria se mantuvo una clara tendencia al alza, sólo interrumpida por los grandes conflictos bélicos, que se acentuó en su segunda mitad, alcanzándose valores medios superiores a las 3.000 kilocalorías, claramente excesivos y causantes de disfunciones.

¿Se observa un cambio similar en España a principios del siglo XX? ¿Converge la ingesta calórica en nuestro país en aquellas fechas con los niveles alcanzados en los países europeos de referencia? Simpson (1989) ha argumentado que, por entonces, España se encontraba en una posición de claro retraso respecto a Europa occidental. Propone para 1900 la cifra de 2.100 kilocalorías/día, estimada a partir de la construcción de las hojas de balance, contemplando una proporción fija de pérdidas. Esta cifra se encuentra claramente por debajo de los países europeos anteriormente señalados, y significaría que nuestro país se mantenía en unos niveles de ingesta calórica tradicionales. Nada que objetar desde el punto de vista metodológico sobre la estimación de Simpson, pero, como han señalado diversos expertos, los resultados pueden estar afectados por la mala calidad de las estadísticas sobre producción agraria, que no la registran de forma adecuada, o por la estimación de unas pérdidas excesivas que pueden tener efectos parecidos sobre los resultados obtenidos. A menudo la producción en huertos familiares o pequeña ganadería doméstica no queda registrada en las estadísticas, y el resto de la producción agraria se estima a la baja. En esta misma dirección, Giral, nutricionista pionero en la introducción en nuestro país del análisis científico de la alimentación que utilizó una metodología basada en las hojas de balance, ya planteaba el carácter aproximado de este tipo de estimaciones, porque "los datos de producción dados por el Servicio Agronómico, son considerados por las mismas personas técnicas que están al frente de este servicio como bastante inferiores a la realidad"7. 


\section{CUADRO 1}

ESTIMACIÓN DE LA INGESTA CALÓRICA MEDIA POR HABITANTE Y DÍA ENTRE 1850 Y 1990

\begin{tabular}{lcccccc}
\hline País & $\mathbf{1 8 5 0}$ & $\mathbf{1 9 0 0}$ & $\mathbf{1 9 3 0}$ & $\mathbf{1 9 6 1}$ & $\mathbf{1 9 9 0}$ & $\mathbf{2 0 0 3}$ \\
\hline Alemania & - & - & 3.040 & 2.889 & 3.310 & 3.484 \\
Francia & 2.480 & 3.192 & 3.127 & 3.194 & 3.284 & 3.623 \\
Italia & 2.466 & 2.682 & 2.520 & 2.914 & 3.591 & 3.674 \\
Gran Bretaña & - & - & 3.110 & 3.290 & 3.267 & 3.450 \\
Bélgica & 2.238 & - & 2.820 & 2.941 & 3.533 & 3.634 \\
España & - & $2.096-2.504$ & $2.426-2.760$ & 2.632 & 3.247 & 3.421 \\
\hline
\end{tabular}

Fuentes: Simpson (1989) para el primer dato de España en 1900 y 1930; Giral (1914) para el segundo de España en 1900; Yates (1960) para el segundo de España en 1930; Toutain (1971) para Francia en 1850, 1900 y 1930; Bekaert (1991) para Bélgica en 1850; Vecchi y Coppola (2006) para Italia en 1861 y 1900; y FAOSTAT para el resto en 1930 y todos en 1961,1990 y 2003.

Razonamientos de este tipo nos llevan a cuestionar la estimación de Simpson de la ingesta calórica media española en 1900, que a continuación analizaremos con detalle. Disponemos de evidencias que refuerzan nuestra opinión de que la ingesta media probablemente era sensiblemente superior a la propuesta por Simpson. Como referencia directa, tenemos la estimación de Giral (1914), sobre lo que él llama "la ración media del español", basada en los datos del Servicio Agronómico, la Asociación de Ganaderos, y las estadísticas de comercio exterior y de la Comisión Extraparlamentaria para la transformación del impuesto de Consumos (CEC). Para cada producto fija un consumo medio a partir de los datos de producción del Servicio Agronómico, descontando las cantidades destinadas a la siembra, y para aquellos productos con flujos importantes con el exterior, añadiendo o restando las importaciones y las exportaciones, respectivamente, a la producción. Para el consumo de carne utiliza las informaciones de la CEC, y para el de leche las estimaciones de la Asociación de Ganaderos. No en todos los casos queda claro si las cifras de consumo son el resultado de la elaboración por parte del autor de las cifras sobre producción que le facilitaba el Servicio Agronómico o si las proporcionaba directamente este Servicio. Aparte de la detracción para la siembra, no consta ninguna deducción por pérdidas. De cualquier forma la metodología parece rigurosa, aunque el autor no deja de insistir en el carácter aproximado de sus estimaciones. Como puede observarse en el Cuadro 2, la "ración media (de energía) del español" se situaría en torno a las 2.500 kilocalorías ${ }^{8}$, claramente por encima de las 2.100 que propone Simpson.

8 Valor calórico estimado a partir de tablas de composición de alimentos actuales, descontando la parte no aprovechable. 
El interés de la propuesta de Giral radica en que complementa este enfoque macro con una serie de estudios de caso: ciudades como Barcelona y Salamanca, colectividades (el regimiento de Albuera, con 249 plazas) o personal recluso 9 , las informaciones de la $\mathrm{CEC}^{10}$ y las encuestas realizadas por el autor en una localidad de Salamanca (Martín del Río), estudiando tres tipos de familias en función de los ingresos. Los resultados, recogidos en el Cuadro 2, son enormemente concluyentes. A excepción del caso de una familia muy pobre de Salamanca, en todos los demás se superan las 2.500 kilocalorías y, en algunos, se llega a las 3.000. Otros ejemplos referidos a casos concretos confirman estos valores de la ingesta calórica. Así, por ejemplo, en el estudio de José Luis Isabel Sánchez sobre la alimentación militar en la segunda mitad del siglo XIX, se reproducen diversos menús cuyo contenido calórico oscila entre 3.500 y 4.000 kilocalorías ${ }^{11}$. En nuestro trabajo sobre la dieta en la comarca catalana del Vallès en la década de 1870, estimábamos la ingesta media en unas 2.800 kilocalorías $^{12}$. Ballesteros (1997) recoge diversas estimaciones sobre el consumo medio de energía en diversas familias de Barcelona, Madrid, Ávila y Vizcaya, entre finales del siglo XIX y principios del XX, que oscilan entre las 2.230 y las 2.760 kilocalorías por persona y día. Ciertamente, estos ejemplos plantean problemas de representatividad, pero es significativo que todos ellos coincidan en una estimación claramente superior a la que propone Simpson.

Una de los motivos principales de que la estimación de la ingesta calórica española de Simpson resulte muy inferior a la europea es el bajo consumo de cereales que asume, significativamente menor que el de los países vecinos. Como expondremos en el próximo apartado, consideramos que su estimación sobre el consumo de pan peca por defecto. Si se aceptan las cifras alternativas sobre el consumo de cereales panificables que proponemos, la ingesta calórica se aproximaría a las 2.500 kilocalorías. En consecuencia, una primera conclusión es que, en el curso de la segunda mitad del siglo XIX, se habría logrado alcanzar una ingesta calórica media de unas 2.500 kilocalorías, cercana a la de Italia y no muy alejada de la de Francia.

9 Los datos proceden del Diccionario de Cadalso, y se corresponden a una de las condiciones de suministro de víveres según disposición de 9 de octubre de 1893. Giral (1914), p. 333.

10 "La información que ha sido escrupulosamente dirigida comprende un total de 423 individuos adultos esparcidos en 73 familias y clasificadas éstas con arreglo a los ingresos pecuniarios de todas clases que ellas tienen". Se establecieron 6 clases. Giral (1914), p. 334.

11 Isabel Sánchez (1994), pp. 107-154.

12 Cussó y Garrabou (2001), pp.26-34. 


\section{CUADRO 2}

EJEMPLOS DE INGESTA CALÓRICA DIARIA A PRINCIPIOS DEL SIGLO XX

\section{Kilocalorías por persona y día}

Ración media del español hacia 1900

Ración media en Barcelona en 1905

Ración media en Salamanca en 1909

Ración media del soldado de caballería

Ración media del recluso

Ración media de CEC (hombre adulto)

Clase 1a (hombre adulto)

Clase $2^{\text {a }}$ (hombre adulto)

Clase $3^{a}$ (hombre adulto)

Clase 4⿳亠丷厂 (hombre adulto)

Clase $5^{\mathrm{a}}$ (hombre adulto)

Clase 6a (hombre adulto)
2.504

2.645

2.733

3.505

2.590

2.896

2.586

2.689

2.760

2.753

3.028

3.562

Familias de Martín del Río (Salamanca)

Ración media de familia muy pobre

Ración media de familia pobre ${ }^{13}$

3.091

Ración media de familia acomodada

3.295

Fuentes: Giral (1914), pp. 349 y 356, y elaboración propia.

\section{Pan, patatas y legumbres: los componentes básicos de la dieta durante la primera fase de la transición nutricional}

En el apartado anterior hemos analizado la primera fase de la transición nutricional, caracterizada por la estabilización y el aumento de la ingesta total. Este último se debió en buena medida al alto consumo de cereales, leguminosas y, sobre todo, patatas, productos que, a finales del Ochocientos, seguían constituyendo la base principal de la ingesta calórica de la población europea occidental, como ejemplifica el caso de Francia en el Cuadro 3.

132.818 según nuestros cálculos, que quedarían en unas 2.500 kilocalorías por miembro de la familia y día si reducimos la ración de patatas que Giral estima en 900 gramos por persona y día. 
¿Cuál era el peso de estos tres productos en la dieta española a principios del siglo XX? Empecemos por el trigo. Simpson fija el consumo aparente de cereales panificables transformados en harina ${ }^{14}$ en 137,7 kilogramos anuales, unos 382 gramos diarios que suponen el 50,6 por 100 de la ingesta calórica total, de los cuales 106,56 corresponden a trigo. De éstos aún habría que deducir unas pérdidas que Simpson estima en un 15 por 100, quedando la cantidad de trigo consumida por habitante y año en unos 90 kilogramos ${ }^{15}$. Como ya hemos indicado anteriormente, consideramos que estas cifras pecan por defecto. Simpson estima el consumo aparente de cereales panificables a partir de la producción media de 1897-1901, le suma el saldo del comercio exterior, le resta la siembra y las pérdidas, y la transforma en harina. Si establecemos el consumo aparente per capita de trigo a partir de las disponibilidades de trigo, y no de harina, obtenemos un resultado de poco más de 142 kilogramos por habitante (véase el Cuadro 5), aún lejos de otras estimaciones, pero que añadidos a los 41,5 de centeno y otros cereales permitirían alcanzar un consumo total de 183,6, es decir, unos 500 gramos de pan por habitante y día; éstos se reducirían nuevamente hasta unos 425 al contemplar unas pérdidas del 15 por 100, que al menos en el caso de los cereales consideramos excesivas ${ }^{16}$. Hemos contrastado estas cifras con otras disponibles para la misma época.

Para el período 1897-1901, las cifras de producción que toma Simpson de las estadísticas de la Junta Consultiva Agronómica arrojan una media anual de unos 30 millones de Qm de trigo, muy cercana a la de 29,5 millones publicada por la CEC para el lapso más amplio, 1896-1906, que utiliza Giral en sus estimaciones y que este autor consideraba calculada a la baja. En la misma línea, Federico Arrazola, a la sazón Director General de Aduanas y autor del informe sobre Producción, comercio y consumo del trigo en España ${ }^{17}$, plantea que "el cálculo de la cosecha de trigo en España es todavía muy imperfecto (...) y está muy distante de la verdad"18. Para el período 18901894, la producción media se estimaba en casi 22,7 millones de Qm, considerando

14 Trigo, centeno, maíz y otros cereales, excluido el arroz.

15 Correspondiendo la diferencia respecto al 53,1 por 100 del Cuadro 3 a la aportación del arroz. Con un consumo de cereales, excluido el arroz, de unos 500 gramos por persona y día, la aportación calórica de los cereales crecería según nuestros cálculos hasta un 60 por 100 del total en 1900, mientras que la aportación proteica de los cereales alcanzaría un 57,6 por 100 del total en la misma fecha.

16 Aceptables probablemente para alimentos frescos como frutas, hortalizas y carne, pero excesivas para alimentos menos perecederos como los cereales, a los que ya se han deducido previamente los desperdicios.

17 Dirección General de Aduanas (1896). Arrazola explica que, ante las malas perspectivas de la cosecha, el Ministerio de Hacienda encargó a sus delegados provinciales que elaborasen informes sobre existencias, perspectivas de la cosecha y necesidades de consumo. Los datos proceden de las respuestas de estos delegados, de la Memoria del inspector general de aduanas de Sitges sobre el comercio de trigo, de la Junta Consultiva Agronómica, de las compañías ferroviarias y de la propia Dirección General de Aduanas. 


\section{CUADRO 3}

PORCENTAJE DE LA INGESTA CALÓRICA DIARIA APORTADO POR TRES GRUPOS DE ALIMENTOS, 1900

\begin{tabular}{lcccc}
\hline País & Cereales & Patatas & Legumbres secas & Total \\
\hline Francia & 58,8 & 8,3 & 2,5 & 69,6 \\
España (Simpson) & 53,1 & 8,2 & 3,1 & 64,4 \\
España (Giral) & 51,4 & 6,8 & 5,9 & 64,1 \\
\hline
\end{tabular}

Fuentes: Toutain (1971), Simpson (1989) y Giral (1914).

\section{CUADRO 4}

PORCENTAJE DE LA INGESTA PROTEICA DIARIA APORTADO POR TRES GRUPOS DE ALIMENTOS, 1900

\begin{tabular}{lcccc}
\hline País & Cereales & Patatas & Legumbres secas & Total \\
\hline Francia & 51,7 & 6,3 & 5,0 & 63,0 \\
España (Simpson) & 54,8 & 9,9 & 7,1 & 71,8 \\
España (Giral) & 50,3 & 6,9 & 12,5 & 69,7 \\
\hline
\end{tabular}

Fuentes: Toutain (1971), Simpson (1989) y Giral (1914).

muchos expertos que la producción real era notablemente superior. Tras analizar los datos sobre las necesidades de siembra, los rendimientos y las cosechas deduce:

"que no se tienen en España datos, no ya seguros, ni siquiera aproximados, del rendimiento de las cosechas de trigo. Todo lo que se aduce afirma el convencimiento de que la producción es muy superior a las cifras oficiales que han podido reunirse, y que, a falta de otras mejores, han debido exponerse"19. Y concluye como "principal objeción que puede hacerse, [que] las cifras de producción resultan aún muy inciertas" 20 .

\footnotetext{
19 Dirección General de Aduanas (1896), p. 24.

20 Dirección General de Aduanas (1896), p. 33.
} 
Admitamos que las cifras de producción que propone Simpson, superiores a las del período 1890-1895 (criticadas por Arrazola), se acercan más a la realidad, aunque siguen persistiendo las opiniones que las consideran demasiado bajas. Las cifras del saldo de comercio exterior no plantean problemas, ni tampoco las de siembra y desperdicios. El resultado obtenido, previo a la transformación del trigo en harina, es, como hemos dicho, 142 kilogramos por habitante y año, que se reducirían a 121 descontadas las pérdidas estimadas por Simpson. Esta última cifra es claramente inferior a las que ofrecen la Comisión Extraparlamentaria (154,2 kilogramos por habitante), la Junta Consultiva Agronómica y el Anuario Estadístico de Cereales $^{21}$, que situaban el consumo de trigo normal por habitante y año a comienzos del siglo XX entre 161 y 163 kilogramos, e incluso el informe de aduanas (123,2 y 136 kilogramos de trigo por habitante y año), como puede verse en el Cuadro $5^{22}$.

Una de las razones, por tanto, que explican los bajos valores de ingesta calórica de Simpson, es el reducido consumo de cereales por habitante y día, que rondaría los 425 gramos, y que nosotros, a partir de las múltiples referencias disponibles, estimamos al menos en 500. En este sentido, Arrazola observa que el consumo aparente que se obtiene de las estadísticas manejadas, 123,2 kilogramos por habitante, es extremadamente bajo, y afirma:

“Admítase, por lo común, que este consumo varia entre 500 y 750 gramos por día y habitante"..."Al tipo de 500 gramos de pan por día, cada habitante debería consumir 182,5 kilogramos al año, equivalentes a 200 kilogramos de trigo"23.

En los años treinta del siglo XIX, Moreau de Jonnés y Madoz proponían un consumo medio de 4 fanegas y 4 celemines, equivalentes a unos 500 gramos diarios, que tomaban de los "economistas españoles del siglo XVIII"24. Posteriormente, diversos autores aceptaron como válida esta cifra, que se convierte en una referencia para una gran parte del siglo, a partir de la cual se calculan las posibilidades de cubrir las necesidades de consumo de diversas zonas de estudio, como la provincia de Girona ${ }^{25}$,

\footnotetext{
$21 \quad$ Morgades (1935), p.15.

22 Germán (2006) ofrece cifras de consumo similares a las recogidas en dicho cuadro para 1890-1894 y para el primer lustro del siglo XX. Para 1896-1900, 129 kilogramos de trigo por habitante y año.

23 Dirección General de Aduanas (1896), p. 40.

24 Moreau de Jonnés (1835), en la versión traducida y adicionada por Madoz, p.140. En la nota 3 Madoz señala "El autor, al fijar el consumo individual de pan en 4 fanegas y 3 celemines, presenta el mínimo de lo que asignan los economistas españoles a cada persona", y añade una relación de autores que proponen cantidades más altas, desde 5 hasta 7 fanegas.

25 Martínez Quintanilla (1865), p. 298.
} 


\section{CUADRO 5}

ESTIMACIONES DEL CONSUMO DE TRIGO A FINALES DEL SIGLO XIX Y PRINCIPIOS DEL XX

Kilogramos por persona y año

$\begin{array}{lc}\text { Simpson (1897-1901) } & 142,0(121) \\ \text { Informe de Aduanas (1890-1904) } & 123,2 \\ \text { Delegados de Hacienda (Informe de Aduanas, 1890-1904) } & 136,0 \\ \text { Comisión Extraparlamentaria (1896-1905) } & 154,2 \\ \text { Junta Consultiva Agronómica (1903) } & 153,6 \\ \text { Junta Consultiva Agronómica (1904) } & 162,1 \\ \text { Junta Consultiva Agronómica (1905) } & 162,1 \\ \text { Anuario Estadístico de Cereales (1901-1902) } & 163,4 \\ \text { Anuario Estadístico de Cereales (1902-1903) } & 163,0 \\ \text { Anuario Estadístico de Cereales (1903-1904) } & 162,6 \\ \text { Anuario Estadístico de Cereales (1904-1905) } & 162,2 \\ \text { Anuario Estadístico de Cereales (1905-1906) } & 161,9\end{array}$

Fuentes: Simpson (1989); Morgades (1935); Junta Consultiva Agronómica (1904), (1905) y (1906); Dirección General de Aduanas (1896) y Comisión Extraparlamentaria (1910).

la comarca de Osona ${ }^{26}$ o la del Vallès ${ }^{27}$. Es interesante comprobar como en las encuestas que la Junta de Agricultura de Barcelona realizó a principios de la década de 1860 sobre producción y consumo de cereales, se fijaba para el partido judicial de Granollers una cifra prácticamente igual, unos 493 gramos $^{28}$ Otras fuentes consultadas avalan también la hipótesis de un consumo de cereales superior al que propone Simpson. Las informaciones antes citadas sobre la dieta del Ejército en los últimos decenios del Ochocientos fijan el consumo de pan en unos 690 gramos diarios ${ }^{29}$. Otra información del Archivo Militar de Segovia fijaba también en 700 gramos la ración de pan a principios del siglo $X^{30}$. Curiosamente una cifra prácticamente igual, unos

26 El autor de una memoria anónima sobre Osona (Estudio agrícola del Llano de Vich. Memoria escrita para optar al premio ofrecido por la Sociedad Barcelonesa de Amigos del País, en su programa del presente año 1872), que con toda probabilidad era J. Salarich, acepta esta misma cifra como referente para calcular las necesidades de consumo de trigo de dicha comarca.

$27 \quad$ Garrabou y Planas (1998).

28 Junta de Agricultura de la provincia de Barcelona, “Estadísticas sobre producción y consumo de cereales. Partido de Granollers", Arxiu Junta de Comerç CXXVI, caixa 164, Biblioteca de Catalunya.

29 Isabel Sánchez (1994), pp. 144-149.

30 Archivo General Militar de Segovia, Sección $2^{\mathrm{a}}$, división $15^{\mathrm{a}}$, legajo $52,42^{\circ} \mathrm{C}$. 
700 gramos diarios, era la cantidad que los ayuntamientos debían proporcionar a las tropas, según una normativa publicada en el Boletín Provincial de la provincia de Barcelona (14 de mayo de 1873) ${ }^{31}$. En los años cincuenta el Ministerio de Gobernación fijaba la misma cantidad para alimentar a la población reclusa ${ }^{32}$. Las cifras ligeramente más altas que aportan estas últimas informaciones son coherentes con el hecho de que se trata del consumo de un adulto masculino con una tasa de actividad alta. Significativamente Cerdà ${ }^{33}$ fijaba el mismo consumo para un obrero adulto en la Barcelona de mediados del siglo XIX. A principios del siglo XX, Alabert (1915) consideraba un consumo medio por persona de 500 gramos diarios, mientras el Anuario de la ciudad de Barcelona lo reducía a unos 360 gramos A partir de las cuentas extraordinariamente precisas sobre compras y menús de la barcelonesa "Escola de Bosc" en la segunda década del siglo XX, estimamos un consumo medio de pan de 307 gramos para los alumnos y 399 para los adultos, y teniendo en cuenta que falta la cena probablemente la ingesta total de pan superaba los 500 gramos $^{34}$ Para principios del siglo XX, Giral (1914) calculaba que la ingesta media en España era de 467 gramos (486,5 con arroz), 630 en el colectivo militar y 575 en la población penal; en las familias de Salamanca oscilaba entre 700 gramos las más pobres, 460 las pobres y sólo 276 las acomodadas. Parece reflejarse la norma de un consumo más alto de cereales en las familias con menos recursos, como estos campesinos salmantinos o la población militar o reclusa, mientras que en las familias acomodadas tiende a ser menor. Finalmente, Lana ${ }^{35}$ confirma unos valores relativamente altos de consumo de cereales para Navarra a principios del siglo XX, al fijarlo en un arco de 182-272 kilogramos por habitante y año (1903-1907), 500-745 gramos diarios, respectivamente. Ciertamente muchas de las evidencias que hemos aportado son de carácter local o se refieren a grupos sociales o instituciones muy distintos, y en consecuencia tienen problemas de representatividad. Sin embargo, el hecho de que prácticamente todas coincidan en unos valores relativamente altos pensamos que avala nuestra hipótesis de que, a principios del siglo XX, y probablemente desde mediados del XIX, el consumo medio de pan en España era significativamente más alto que el considerado por Simpson, y se situaría alrededor de 500 gramos, una cantidad muy similar a la de Francia e Italia a comienzos del Novecientos. ${ }^{36}$

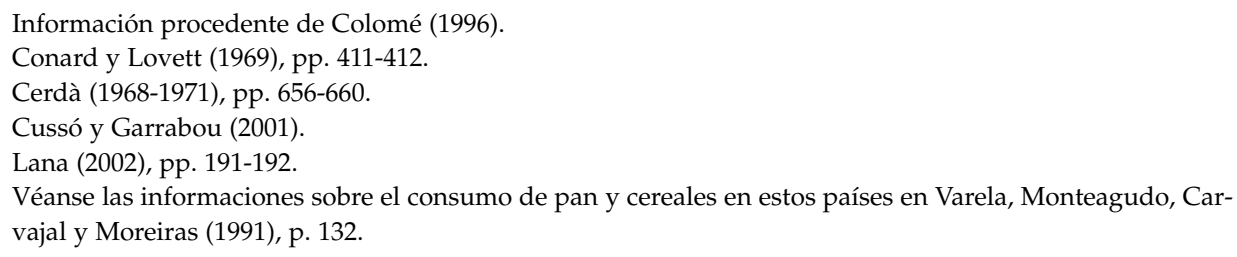


La incorporación de la patata en la dieta fue también uno de los cambios más importantes que experimentó el sistema alimentario español del siglo XIX. Posibilitó disponer de calorías baratas y, probablemente, es uno de los factores determinantes del alza de la ingesta calórica media de finales del Ochocientos. El cultivo de la patata se había difundido ampliamente en Galicia desde el siglo XVIII, pero en el resto de la península no empezó a popularizarse hasta después de las guerras napoleónicas. A mediados de XIX, su consumo ya se había extendido considerablemente tanto en las zonas rurales como en las urbanas. Para principios del XX, Simpson propone unos 105 kilogramos anuales, unos 290 gramos de consumo medio diario, por encima de los 236 de Giral. Este mismo autor en sus encuestas sobre la localidad salmantina fija en 300 gramos el consumo de patatas para las familias muy pobres y en 900 para las pobres, en tanto que las acomodadas prácticamente no las consumían. En nuestro trabajo sobre el Vallès considerábamos una cifra más alta, unos 169 kilogramos, que representan unos 460 gramos diarios; Cerdà, para la ciudad de Barcelona, propone unos 125 kilogramos (350 gramos diarios). En los menús del Ejército, el consumo de patatas oscilaba entre 280, 460, 590 y 690 gramos, constando en alguno de ellos hasta 800 gramos diarios. A principios del siglo XX, para la ciudad de Barcelona, Alabert lo fijaba en 100 gramos por habitante y los anuarios de Barcelona entre 112 y 128.

La importancia de la consolidación del consumo de patatas en la dieta española durante el siglo XIX fue muy notable. Como recoge el Cuadro 3, dicho tubérculo proporcionaba en 1900 cerca del 10 por 100 de la ingesta energética total, y al tratarse de calorías baratas resultó decisivo para que las clases con menos recursos pudieran incorporarse al proceso de regularización y aumento de la ingesta calórica que se estaba imponiendo en la mayoría de los países europeos. Es significativo que la ingesta de patatas también fuese muy alta en la dieta de las clases populares, del Ejército o de la población reclusa, y disminuyese a medida que los ingresos eran más altos.

Finalmente, el consumo de legumbres sería de 9,69 kilogramos por persona y año, unos 26,5 gramos diarios, según las estimaciones de Simpson. Giral prácticamente dobla esta cifra, fijando en 17 kilogramos (unos 47 gramos) el consumo medio español. En nuestro trabajo sobre el Vallès proponíamos una cifra más elevada, 74 gramos diarios. También otras fuentes dan valores más altos. Cerdà calculaba cerca de 100 gramos diarios y en los menús militares las legumbres variaban desde 72-115 gramos hasta 300. Según Alabert (1915), el consumo medio de legumbres en Barcelona era de 50 gramos por persona, mientras el anuario estadístico de la ciudad de Barcelona lo reducía a unos 38 en las primeras décadas del siglo XX. Mucho mayor era dicho consumo, por las mismas fechas, en Vizcaya, fijándose en 147 gramos. Probablemente, la estimación de Simpson también peca en este caso por defecto y debiera revisarse al alza. De todos modos, aún aceptando sus cifras se comprueba la con- 
siderable importancia que continuaban teniendo las legumbres en la dieta de los españoles de principios del siglo XX. Sus aportaciones en proteínas de alto valor biológico, complementarias de las de los cereales, seguían siendo decisivas.

En definitiva, se puede afirmar que en la España de principios del siglo XX se había alcanzado una ingesta aparente de energía que cubriría las necesidades medias estimadas de la población en aquel momento, entre 2.250 y 2.300 kilocalorías por habitante y día ${ }^{37}$. Pese a los progresos registrados durante el siglo XIX, el consumo de la población española sería todavía inferior al de otras poblaciones de Europa occidental. Los cereales representarían más del 50 por 100 de la energía ingerida, las patatas cerca de un 10, y las legumbres entre un 3 y un 6 por 100. En total los tres grupos de alimentos aportarían casi dos tercios de la energía ingerida, proporciones parecidas a las que presentaba Francia en esas mismas fechas (véase el Cuadro 3).

\section{La transición nutricional hasta mediados del siglo $\mathrm{XX}$}

Es a principios del siglo XX, sobre todo después de la I Guerra Mundial, cuando se observan transformaciones importantes en los sistemas alimentarios europeos. Por un lado, la ingesta calórica registra una tendencia moderada al alza, y, por otro, se produce la progresiva sustitución de una dieta basada fundamentalmente en cereales, patatas y legumbres — que habían aportado tradicionalmente un alto porcentaje de las calorías y nutrientes - por otra en la que las substancias animales, carne, leche, huevos, pescado y determinadas grasas animales adquieren una creciente importancia (véase el Cuadro 6). El consumo de cereales y, en ciertos casos, el de patatas, empezaron a descender de forma moderada hasta la II Guerra Mundial, mientras que el consumo de legumbres quedó reducido a una posición prácticamente marginal.

¿Se observan cambios similares en España en el primer tercio del siglo XX? Como se observa en el Cuadro 1, probablemente la ingesta total había mejorado en la década de 1930, alcanzando valores entre las 2.400 y las 2.700 kilocalorías. Pero la composición de la dieta y la evolución del consumo aparente de cereales, patatas y legumbres, no coinciden plenamente con lo sucedido en otros países europeos (véanse los Cuadros 7 y 8 ).

Respecto a las patatas, disponemos de las estimaciones de Simpson para 1930 y de la serie de García Barbancho, que empieza en 1926. A partir de estos datos, parece deducirse que, aún en el contexto de la primera fase de la transición nutricional

37 Cussó (2001) y (2005). 


\section{CUADRO 6}

CONSUMO POR HABITANTE DE ALGUNOS ALIMENTOS SELECCIONADOS EN EUROPA OCCIDENTAL, PRIMERA MITAD DEL SIGLO XX

\begin{tabular}{lcccccc}
\hline Kilogramos/año & \multicolumn{2}{c}{ Cereales (harina) } & \multicolumn{2}{c}{ Carne } & \multicolumn{2}{c}{ Leche } \\
\hline & $\mathbf{1 9 1 0 - 1 9 1 4}$ & $\mathbf{1 9 3 4 - 1 9 3 8}$ & $\mathbf{1 9 1 0 - 1 9 1 4}$ & $\mathbf{1 9 3 4 - 1 9 3 8}$ & $\mathbf{1 9 1 0 - 1 9 1 4}$ & $\mathbf{1 9 3 4 - 1 9 3 8}$ \\
\hline Bélgica & 135,0 & 114,4 & 29,5 & 47,2 & 65,5 & 79,8 \\
Francia* $_{\text {Alemania }}^{185,0}$ & 123,7 & 33,6 & 55,2 & 100,0 & 85,5 \\
Gran Bretaña & 127,7 & 113,0 & 48,2 & 52,8 & 133,0 & 138,6 \\
& 95,0 & 95,3 & 35,5 & 62,6 & 70,1 & 107,3 \\
\hline
\end{tabular}

* 205,1 y 134,4 kilogramos de harina para 1905-1913 y 1935-1938, respectivamente, según Toutain (1971).

\section{CUADRO 7}

PORCENTAJE DE LA INGESTA CALÓRICA DIARIA APORTADO POR TRES GRUPOS DE ALIMENTOS, 1930

\begin{tabular}{lcccc}
\hline País & Cereales & Patatas & Leguminosas & Total \\
\hline Francia & 43,2 & 10,9 & 2,3 & 56,4 \\
España & 45,8 & 13,3 & 3,6 & 60,7 \\
\hline
\end{tabular}

Fuentes: Toutain (1971) y Simpson (1989).

\section{CUADRO 8}

PORCENTAJE DE LA INGESTA PROTEICA DIARIA APORTADO POR TRES GRUPOS DE ALIMENTOS, 1930

\begin{tabular}{lcccc}
\hline País & Cereales & Patatas & Leguminosas & Total \\
\hline Francia & 38,4 & 8,4 & 4,8 & 51,6 \\
España & 47,4 & 14,0 & 6,7 & 68,2 \\
\hline
\end{tabular}

Fuente: Toutain (1971) y Simpson (1989). 


\section{GRÁFICO 1}

CONSUMO APARENTE DE PATATAS EN ESPAÑA, 1900-2000

(kilogramos por persona y año)

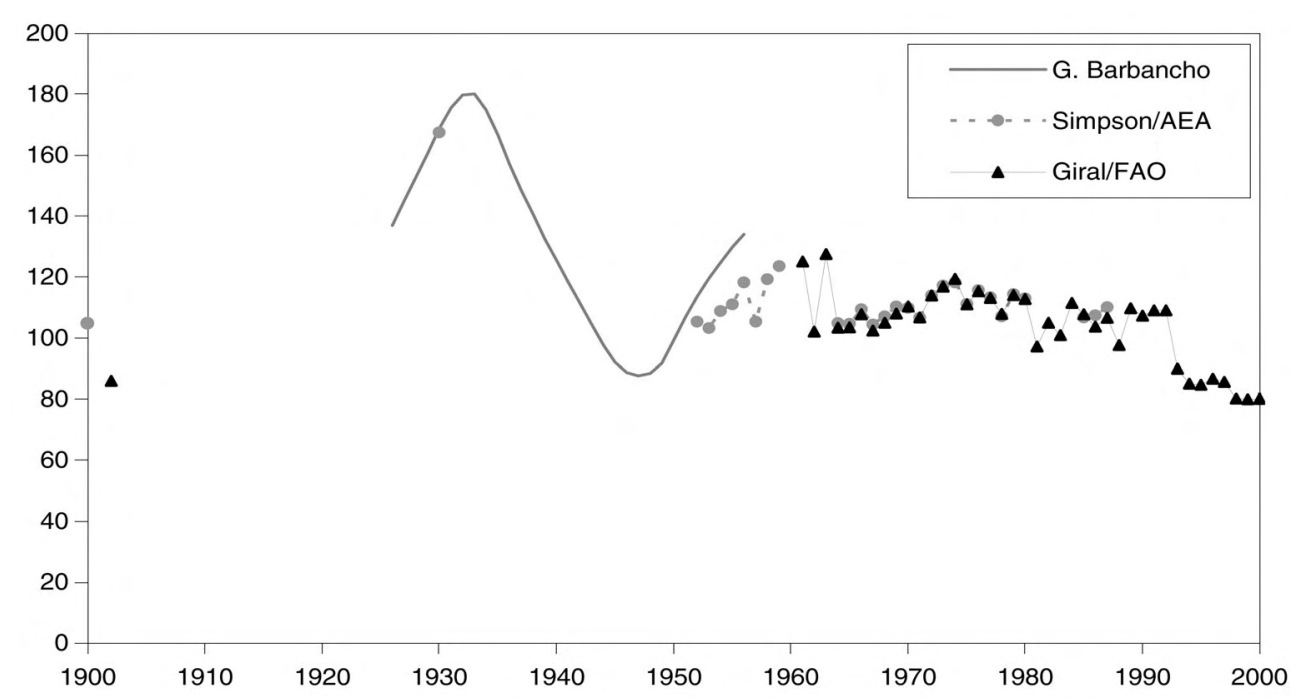

Fuentes: Simpson (1989), para 1900 y 1930; Morgades (1935), para 1902-1935; Giral (1914), para 1900; Anuario de Estadística Agraria (AEA) a partir de 1952; García Barbancho (1960a) y (1960b), para 1926-1956, y FAOSTAT a partir de 1961.

moderna, se produce en estas décadas un notable aumento del consumo de patatas, que compensaría con creces una moderada reducción del de cereales y legumbres (como se puede apreciar en los Gráficos 1, 2 y 3), manteniéndose e, incluso, aumentando la aportación energética y de hidratos de carbono de estos tres grupos de alimentos al total. En concreto, el consumo de patatas, que rondaba los 100 kilogramos en 1900, alcanza cerca de 180 (500 gramos) en la década 1930. Los datos de legumbres no son concluyentes: oscilan entre los casi 10 kilogramos anuales de Simpson y los 17 de Giral a comienzos del siglo, se situarían en unos 12,5 en los años veinte, según las estimaciones de García Barbancho, y se reducirían ligeramente, hasta 11,5, en la década de 1930, suponiendo menos del 5 por 100 de la ingesta calórica total en las estimaciones más optimistas. El consumo de cereales en la década de 1920 se situaría, según García Barbancho, que sólo contabiliza trigo y centeno, en 167 kilogramos por habitante y año, descendiendo hasta 160 en la de 1930, claramente por encima de los 133,9 que propone Simpson, pero que sin convertir en harina alcanzarían prácticamente los 180 kilogramos, contabilizando trigo, centeno y maíz. 


\section{GRÁFICO 2}

CONSUMO APARENTE DE CEREALES EN ESPAÑA, 1900-2000

(kilogramos por persona y año)

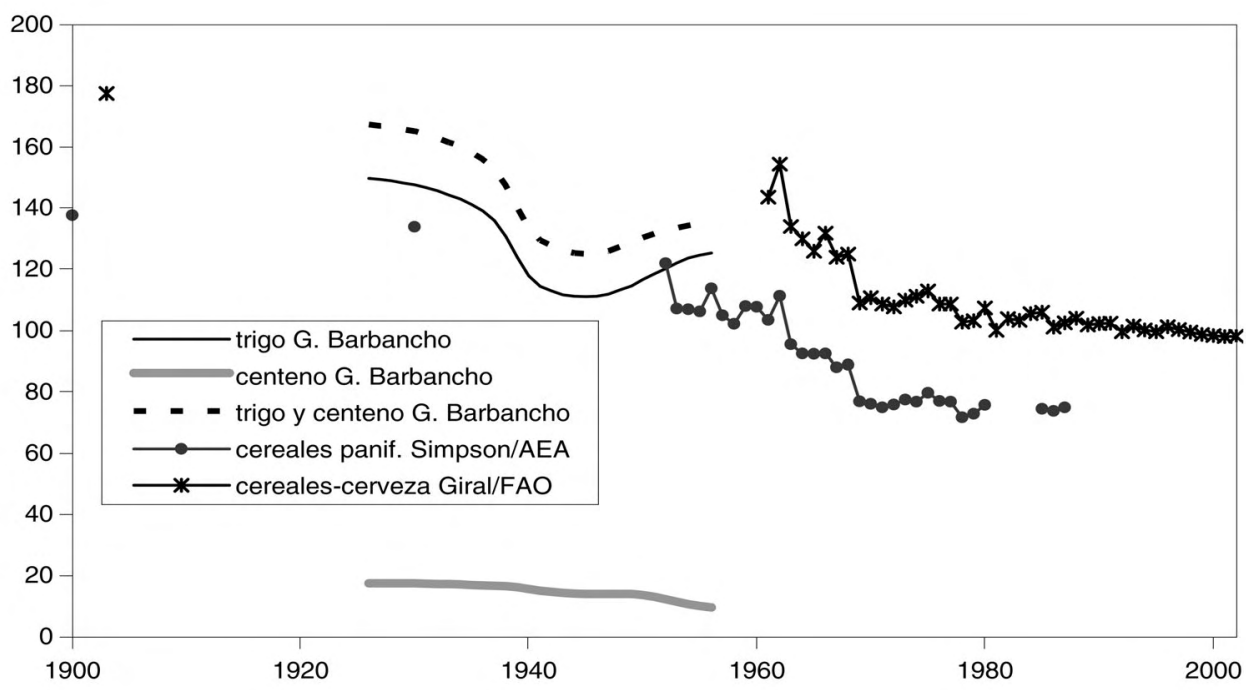

Fuentes: Las mismas del Gráfico 1.

GRÁFICO 3

CONSUMO APARENTE DE LEGUMBRES EN ESPAÑA, 1900-2000

(kilogramos por persona y año)

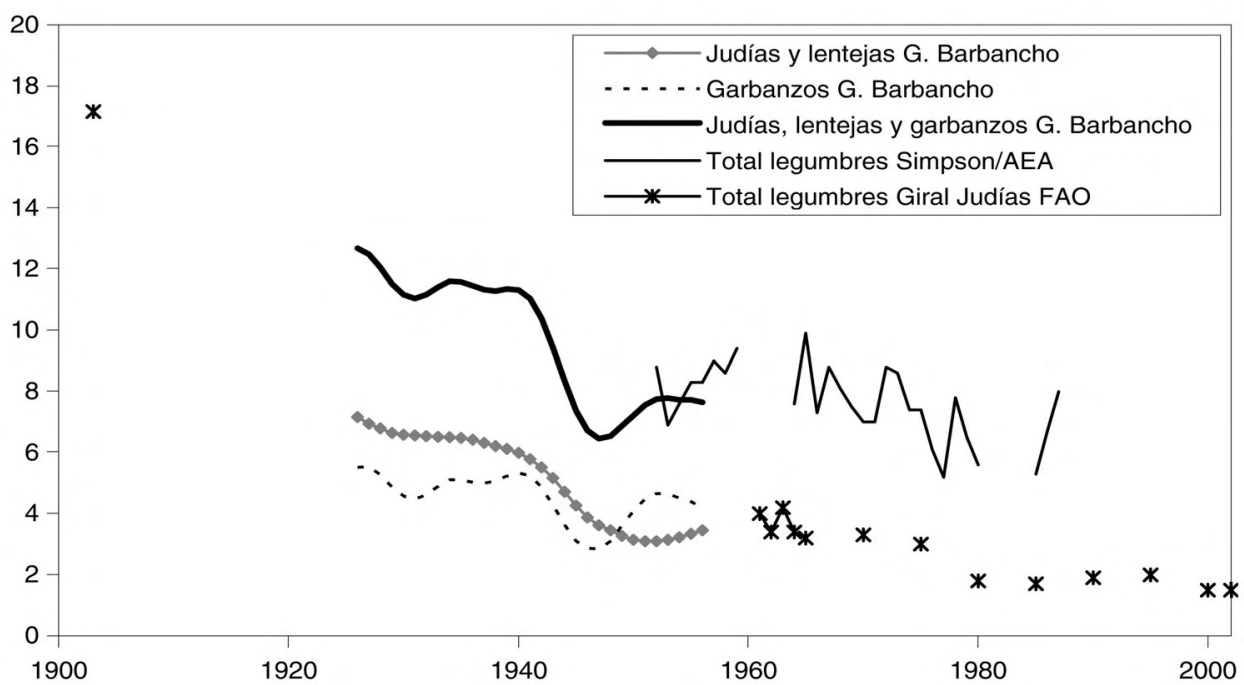

Fuentes: Las mismas del Gráfico 1. 
¿Disminuyó en España el consumo de pan en el primer tercio del siglo XX como en Francia, donde en la década de 1930 se había reducido a 325 gramos, o se mantuvo como en Italia? Aunque algunos contemporáneos ${ }^{38}$ sostuvieron que el consumo de pan se había reducido en las grandes ciudades como Barcelona, a consecuencia de los cambios de los hábitos alimentarios impuestos por la industrialización, no existen evidencias estadísticas claras de que se hubiera producido una disminución significativa (véase el Gráfico 2). A las cifras dadas anteriormente se podrían añadir las aportadas por algunos diputados en un debate de la Asamblea Nacional: mientras uno afirmó como un hecho aceptado que el consumo de pan era de 300 gramos, en la misma sesión otro señaló que era de 493, sin que cuestionara la cifra dada por el primero $^{39}$. Las cifras recogidas en el Anuario Estadístico de Cereales reflejan un suave descenso del consumo normal de trigo por habitante, que pasa de 163 kilogramos al iniciarse el siglo, a unos 156 a comienzos del decenio de 1920 y a 151 en la primera mitad del de $1930^{40}$. No obstante, el cambio más importante que se produjo en el primer tercio del siglo XX fue la generalización del pan blanco, que desplazó al pan moreno elaborado con cereales distintos del trigo y, sobre todo, con harinas de alta extracción con un contenido elevado de salvado.

Alcanzado el primer tercio del siglo XX, la ingesta calórica media de la población española se ha incrementado, si bien sigue por debajo de la de los países más desarrollados de Europa occidental. Ha aumentado en términos absolutos la aportación de los tres grupos de alimentos estudiados, especialmente de las patatas, aunque su aporte conjunto en términos relativos se ha reducido debido al incremento del consumo más que proporcional de otros alimentos, entre los que destaca, por su relevante aportación de energía y nutrientes a la dieta, el moderado aumento de los alimentos de origen animal (véanse los Cuadros 9 y 10) ${ }^{41}$.

En Francia, según las estimaciones realizadas por Toutain (1971), la aportación de las patatas también crece sensiblemente, hasta los 175-178 kilogramos por persona y año (nivel similar al de España), cayendo la de los cereales (pan o harina); la de las legumbres, que no alcanzan los 7 kilogramos por persona y año ${ }^{42}$; y la del conjunto de los tres grupos, tanto en términos absolutos como relativos. Los datos mane-

\footnotetext{
$38 \quad$ Véase, por ejemplo, Falgueras (1930), p. 7.

39 "El problema del trigo, de la harina y del pan en la Asamblea Nacional", extracto del diario de sesiones de la Asamblea Nacional, sesión plenaria del 30 de octubre de 1928, reproducido en Molinería y Panadería, Barcelona, 30 de noviembre de 1928, pp. 983-994.

Morgades (1935), p. 15.

41 Como se puede comprobar en las cifras presentadas por Simpson (1989) y en muchas otras referencias locales, regionales o nacionales. Véanse, entre otras, las recientemente recogidas en Nicolau y Pujol (2005), y Cussó y Garrabou (2006).

42 4,3 kilogramos por persona y año. Datos de la FAO recogidos en Hercberg, Rouaud y Dupin (1988).
} 


\section{CUADRO 9}

PORCENTAJE DE LA INGESTA CALÓRICA DIARIA DE LA POBLACIÓN ESPAÑOLA APORTADO POR ALIMENTOS DE ORIGEN ANIMAL, 1900 Y 1930

\begin{tabular}{lcccc}
\hline Año & Carne & Leche & Pescado & Total \\
\hline $\mathbf{1 9 0 0}$ & 5,6 & 3,0 & 1,3 & 11,0 \\
$\mathbf{1 9 3 0}$ & 8,0 & 4,5 & 1,2 & 14,2 \\
\hline
\end{tabular}

Fuente: Simpson (1989).

CUADRO 10

PORCENTAJE DE LA INGESTA PROTEICA DIARIA DE LA POBLACIÓN ESPAÑOLA APORTADO POR ALIMENTOS DE ORIGEN ANIMAL, 1900 Y 1930

\begin{tabular}{lcccc}
\hline Año & Carne & Leche & Pescado & Total \\
\hline $\mathbf{1 9 0 0}$ & 7,2 & 3,5 & 9,2 & 22,9 \\
$\mathbf{1 9 3 0}$ & 8,2 & 6,9 & 8,1 & 25,1 \\
\hline
\end{tabular}

Fuente: Simpson (1989).

jados por la FAO y la OECE para el período 1934-1938 para países como Alemania y Bélgica, muestran un alto consumo de patatas - 187 y 156 kilogramos por habitante y año, respectivamente-, similar al de España, y un consumo de cereales y de legumbres sensiblemente más bajo a las ingestas de la población española ${ }^{43}$. Por otra parte, en Europa occidental el consumo de productos de origen animal progresa más rápidamente que en España, constituyendo éste uno de los aspectos más representativos del moderado retraso hispano en la transición nutricional moderna.

La depresión de los años 30 y, especialmente, los años de la Guerra Civil y la larga y dramática posguerra, rompen la tendencia de las primeras décadas del siglo, operándose una drástica reducción del consumo de estos alimentos básicos y, en 
general, un grave deterioro de la alimentación de gran parte de la población española. Hasta finales de la década de 1940 no se inicia la recuperación del consumo, que en ningún caso parece alcanzar los niveles de la primera mitad de la década de 1930. El consumo de legumbres y patatas se reduce prácticamente a la mitad los peores años, en tanto que la reducción de las disponibilidades de cereales llegará al 25 por 100 (véanse los Gráficos 1, 2 y 3). El aumento de las disponibilidades de batata, boniatos y otras hortalizas solo podrá paliar muy parcialmente el hundimiento de los alimentos básicos. Otras referencias como el racionamiento y su precario cumplimiento confirman la magnitud del retroceso experimentado. El Cuadro 11, por ejemplo, recoge las raciones tipo para un hombre adulto fijadas en junio de 1939.

Para las mujeres adultas y los hombres mayores de 60 años, las raciones establecidas eran un 80 por 100 de las anteriores, y para los niños hasta 14 años un 60 por 100. Si consideramos un promedio nacional en torno al 80 por 100 de la ración tipo, se puede apreciar que el consumo de pan o patatas de la parte de la población condenada a sobrevivir con el racionamiento se situaba sensiblemente por debajo de las disponibilidades estimadas, siendo superiores sólo las raciones de legumbres, en el caso de haberse cumplido. Pero durante gran parte del período la situación fue mucho peor. En los primeros años de posguerra, a causa de las malas cosechas y el aislamiento exterior, no había alimentos suficientes ni para cubrir el racionamiento establecido. En el caso del pan, por ejemplo, la cantidad suministrada llegó a situarse en torno a 150 gramos por persona y día ${ }^{44}$. A escala nacional, para el resto de alimentos racionados y, en concreto, para patatas y legumbres, las cantidades realmente distribuidas por habitante y año apenas representaban, en el mejor de los casos, una tercera parte de las estipuladas, quedando las posibilidades de supervivencia a expensas del mercado negro en las zonas urbanas y del autoconsumo en las rurales.

\section{Cambios en los patrones de consumo en España en la segunda mitad del siglo XX}

A finales de la década de 1940, superada la depresión económica del decenio precedente y, sobre todo, la II Guerra Mundial y los primeros años de posguerra, se produce la recuperación definitiva del consumo de alimentos y se reemprende el proceso de transición nutricional moderna en Europa. Como puede verse en el Cuadro 1, la ingesta calórica se recuperó a principios de la década de 1960 y registra un creci-

\footnotetext{
$44 \quad$ Alburquerque (1981), p. 427.
} 


\section{CUADRO 11}

RACIONES TIPO PARA UN HOMBRE ADULTO, JUNIO DE 1939

\begin{tabular}{lr}
\hline Alimentos & Gramos \\
\hline Pan & 400 \\
Patatas & 250 \\
Legumbres secas* & 100 \\
Aceite & 50 \\
Café & 10 \\
Azúcar & 30 \\
Carne & 125 \\
Tocino & 25 \\
Bacalao & 75 \\
Pescado fresco & 200 \\
\hline * Garbanzos, judías, lentejas y arroz. \\
\hline Fuentes: Alburquerque (1981). \\
\hline
\end{tabular}

\section{CUADRO 12}

CANTIDADES MEDIAS DISTRIBUIDAS EN RÉGIMEN DE RACIONAMIENTO, 1941-1949 (kilogramos por habitante y año)

\begin{tabular}{ccc}
\hline Años & Legumbres & Patatas \\
\hline $\mathbf{1 9 4 1}$ & 3,06 & - \\
$\mathbf{1 9 4 2}$ & 2,88 & 13,13 \\
$\mathbf{1 9 4 3}$ & 3,21 & 17,94 \\
$\mathbf{1 9 4 4}$ & 2,85 & 22,23 \\
$\mathbf{1 9 4 5}$ & 2,11 & 21,83 \\
$\mathbf{1 9 4 6}$ & 2,28 & 14,95 \\
$\mathbf{1 9 4 7}$ & 3,19 & 18,17 \\
$\mathbf{1 9 4 8}$ & 3,22 & 23,58 \\
$\mathbf{1 9 4 9}$ & 3,63 & 28,63 \\
\hline
\end{tabular}

Fuentes: Molinero e Ysàs (1985). 
miento moderado hasta finales del siglo, en que se estabiliza claramente por encima de las 3.000 kilocalorías. Por otra parte, prosigue durante esta etapa de la transición alimenticia en los países europeos la disminución del consumo de cereales, patatas y legumbres, sustituidos por productos de origen animal y, en menor medida, por vegetales frescos y frutas. En España se sigue una evolución similar, aunque se mantiene inicialmente un moderado retraso. Los datos de 1960 muestran el alcance de la recuperación a partir de esta fecha, con un crecimiento a un ritmo superior al europeo que sobrepasa ampliamente las 3.000 kilocalorías y se sitúa a niveles similares a los europeos de referencia ${ }^{45}$. En lo que atañe a los alimentos objeto de nuestro estudio, en España se desarrolla una primera fase de crecimiento del consumo, sin alcanzar los niveles del primer tercio del siglo, y una segunda de prolongado descenso hasta la actualidad, que presenta una gran similitud con la evolución de su consumo en otros países desarrollados, en un contexto de clara convergencia hacia los sistemas alimentarios de los países de Europa noroccidental, y de progresivo alejamiento de los patrones tradicionales de la dieta mediterránea ${ }^{46}$. Efectivamente, como se puede apreciar en los Cuadros 13 a 18, elaborados a partir de los datos de las hojas de balance recogidas por FAOSTAT, la aportación energética y proteica de cereales y patatas en España, que aún en 1960 era conjuntamente muy superior a la de países de Europa noroccidental, como Alemania o Francia, se reduce hasta 1990 en unos 20 puntos, situándose en niveles similares a los de los países que habían sido pioneros en el proceso de transición alimenticia. Sólo en Italia, debido a la mayor importancia de los cereales y el papel marginal de la patata en la dieta ${ }^{47}$, la aportación nutritiva (energía y proteínas) de estos grupos de alimentos representa aún poco más de una tercera parte del total.

Así, el consumo de patatas en España rondaba los 100 kilogramos por persona y año en 1950, superando los 125 una década más tarde. Desde entonces, el consumo se estabilizó en torno a 100-110 kilogramos hasta principios de los 90, cuando se produjo un descenso, hasta hoy definitivo, situándose en 80 kilogramos por habitante y año. Los datos obtenidos de las encuestas de presupuestos familiares y de consumo, realizadas desde los años sesenta, confirman la tendencia descrita. El consumo en 1964 era de 109 kilogramos por habitante y año, de 72 en 1981, y de 53 en 1991.

\footnotetext{
45 Ligeramente por encima de las 3.000 kilocalorías en 1964, y 2.634 en 1991, según las encuestas de presupuestos familiares de sendos años.

46 De los que conserva un superior consumo de pescado, de grasas vegetales (aceite de oliva), frutas y verduras frescas, y un mínimo consumo de legumbres. Véanse Abad, García Delgado y Muñoz (1994) y Varela (1995).

47 Cabe recordar que el contenido de energía y proteínas por unidad de peso de los cereales es muy superior al de las patatas.
} 


\section{CUADRO 13}

PORCENTAJE DE LA INGESTA CALÓRICA DIARIA APORTADO POR DOS GRUPOS DE ALIMENTOS, 1961

\begin{tabular}{lccc}
\hline País & Cereales & Patatas & Total \\
\hline Alemania & 24,9 & 8,8 & 33,7 \\
España & 40,2 & 8,7 & 48,9 \\
Francia & 29,7 & 6,6 & 36,3 \\
Italia & 44,7 & 3,4 & 48,1 \\
Reino Unido & 23,7 & 5,5 & 29,2 \\
\hline
\end{tabular}

Fuentes: Elaboración propia a partir de FAOSTAT.

CUADRO 14

PORCENTAJE DE LA INGESTA PROTEICA DIARIA APORTADO POR DOS GRUPOS DE ALIMENTOS, 1961

\begin{tabular}{lccc}
\hline País & Cereales & Patatas & Total \\
\hline Alemania & 26,8 & 7,5 & 34,3 \\
España & 42,4 & 7,0 & 49,4 \\
Francia & 29,7 & 4,8 & 34,6 \\
Italia & 48,5 & 2,4 & 51,0 \\
Reino Unido & 26,6 & 4,4 & 31,0 \\
\hline
\end{tabular}

Fuentes: Elaboración propia a partir de FAOSTAT.

Por lo que respecta a las legumbres, la recuperación llega, al menos, hasta mediados de la década de 1960, con un consumo de 10 kilogramos por habitante y año según los Anuarios de Estadística Agraria (próximo a los 15, según la encuesta de presupuestos familiares de 1964), para luego descender progresivamente. A principios de los 90, el consumo se había reducido a la mitad, unos 7,4 kilogramos según la encuesta de presupuestos familiares, un 2,4 por 100 de la ingesta calórica total, claramente superior aún al consumo de los países europeos (1 kilogramo al año $)^{48}$. 


\section{CUADRO 15}

PORCENTAJE DE LA INGESTA CALÓRICA DIARIA APORTADO POR DOS GRUPOS DE ALIMENTOS, 1990

\begin{tabular}{lccc}
\hline País & Cereales & Patatas & Total \\
\hline Alemania & 21,9 & 4,4 & 26,4 \\
España & 23,1 & 6,0 & 29,1 \\
Francia & 23,5 & 3,7 & 27,2 \\
Italia & 31,8 & 2,1 & 33,9 \\
Reino Unido & 22,2 & 5,9 & 28,1 \\
\hline
\end{tabular}

Fuentes: Elaboración propia a partir de FAOSTAT.

\section{CUADRO 16}

PORCENTAJE DE LA INGESTA PROTEICA DIARIA APORTADO POR DOS GRUPOS DE ALIMENTOS, 1990

\begin{tabular}{lccc}
\hline País & Cereales & Patatas & Total \\
\hline Alemania & 22,4 & 3,5 & 25,8 \\
España & 22,5 & 4,4 & 26,9 \\
Francia & 21,7 & 2,7 & 24,4 \\
Italia & 31,9 & 1,6 & 33,5 \\
Reino Unido & 24,4 & 4,9 & 29,3
\end{tabular}

Fuentes: Elaboración propia a partir de FAOSTAT.

CUADRO 17

PORCENTAJE DE LA INGESTA CALÓRICA DIARIA APORTADO POR DOS GRUPOS DE ALIMENTOS, 2000

\begin{tabular}{lccc}
\hline País & Cereales & Patatas & Total \\
\hline Alemania & 23,1 & 4,1 & 27,2 \\
España & 21,4 & 4,2 & 25,6 \\
Francia & 24,2 & 3,3 & 27,5 \\
Italia & 31,1 & 1,9 & 33,0 \\
Reino Unido & 24,5 & 6,5 & 31,0
\end{tabular}

Fuente: elaboración propia a partir de FAOSTAT. 
CUADRO 18

PORCENTAJE DE LA INGESTA PROTEICA DIARIA APORTADO POR DOS GRUPOS DE ALIMENTOS, 2000

\begin{tabular}{lccc}
\hline País & Cereales & Patatas & Total \\
\hline Alemania & 24,9 & 3,3 & 28,2 \\
España & 20,4 & 3,1 & 23,5 \\
Francia & 22,5 & 2,4 & 24,9 \\
Italia & 30,8 & 1,4 & 32,2 \\
Reino Unido & 26,4 & 5,1 & 31,5 \\
\hline
\end{tabular}

Fuente: elaboración propia a partir de FAOSTAT.

El consumo de cereales se recuperó hasta entrada la década de 1950, iniciando un notable descenso en la siguiente, que se moderó en las posteriores. Varela, Monteagudo, Carvajal y Moreira (1991) analizan exhaustivamente el drástico cambio que se operó en dicho consumo a partir de los años sesenta. Cabe destacar el gran retroceso que experimentó el de pan blanco, compensado un poco dentro del grupo de los cereales por el aumento del consumo de pan integral y bollería, claro reflejo de la disminución de la demanda de "transformados tradicionales" y del aumento de la de "alimentos procesados", tal y como son definidos por Abad, García Delgado y Muñoz $(1994)^{49}$. Las encuestas de presupuestos familiares corroboran esta evolución: de 159 kilogramos por habitante y año en 1964 (134 en forma de pan), se pasó a 99 en 1981 y a 87,2 en 1991 (59,5 en forma de pan, de ellos sólo 50 de pan blanco).

\section{A modo de conclusión: el impacto nutricional de los cambios en los patrones de consumo}

Como ha quedado dicho, las últimas décadas del siglo XIX y el primer tercio del XX se caracterizan por la regularización y el aumento del suministro de nutrientes, especialmente de energía, produciéndose con ello una progresiva mejora del estado nutritivo de la población española, sin alcanzar, no obstante, los niveles de otros países de Europa occidental. La evolución positiva en este lapso de indicadores como la mortalidad, sobre todo la infantil y la juvenil —ligadas aún en gran parte, directa e 
indirectamente, a la alimentación-, o la talla media de los reclutas españoles, pueden atribuirse en buena medida a la mejora del estado nutricional de la población ${ }^{50}$.

Sin embargo, debe considerarse que nos movemos con valores medios; dada la desigual distribución real de los nutrientes entre la población, es probable que una parte notable de ésta siguiese consumiendo cantidades sensiblemente inferiores a la media y a sus necesidades, permaneciendo expuesta a la malnutrición. En este sentido, aplicando a la estimación de la ingesta energética media de la población española de Giral, o a la calculada por Simpson en 1900 corregida al alza —unas 2.500 kilocalorías por habitante y día, una distribución real de la energía ingerida como las estimadas por Fogel ${ }^{51}$ para Francia y para Inglaterra a finales del XVIII ${ }^{52}$, obtendríamos que al menos un 30 por 100 de la población ingeriría cantidades de energía claramente inferiores a sus necesidades. Peor incluso era la situación en cuanto a numerosos micronutrientes, como el calcio o la vitamina A, fundamentales para el desarrollo físico y el correcto funcionamiento del sistema inmunológico. Algo menos dramáticos en términos calóricos, aunque igualmente graves para otros nutrientes, parecen ser los desequilibrios en la distribución derivada de las encuestas utilizadas por Giral (véase el Cuadro 2).

Desde mediados de la década de 1930 y hasta la de 1950 se interrumpe el proceso anteriormente descrito y se produce un grave deterioro del estado nutritivo de la población española, también apreciable en los indicadores complementarios señalados. La ingesta de nutrientes se reduce como resultado de la combinación entre la caída de la producción de alimentos, especialmente la de productos básicos como los cereales, los problemas en su distribución y el difícil acceso al suministro exterior, provocada por la Guerra Civil, la larga posguerra y la II Guerra Mundial.

En los años cincuenta se retoma el proceso interrumpido en los treinta, apreciándose una notable mejora del estado nutritivo de la población, que alcanza en las últimas décadas del siglo XX los niveles de los países más desarrollados de Europa occidental. Inicialmente se registra un aumento de la ingesta de energía y nutrientes proporcionados por alimentos básicos como los cereales o las patatas, fruto de la recuperación de su producción y consumo. Pero dicho aumento pronto deja paso a un estancamiento y posterior reducción de la aportación nutritiva de estos alimentos básicos, que es compensada por un gran aumento del consumo de productos de origen animal — que pasan de aportar en 1930 sólo el 14 por 100 de la energía a pro-

\footnotetext{
50 Véanse sobre la evolución de estos indicadores en el período aquí analizado: Gómez Mendoza y Pérez Moreda (1985); Coll y Quiroga (1994); Martínez Carrión (1991) y (1994); Martínez Carrión y Pérez Castejón (1998); Quiroga (2001); Cussó y Nicolau (2000), y Cussó (2001), entre otras obras.

51 Consideradas más igualitarias que las observadas actualmente en diversos países del Tercer Mundo. Fogel (1993).

52 Simpson (1997), p. 377, utiliza para 1900 la distribución presentada por Fogel para la Inglaterra de finales del siglo XVIII.
} 
porcionar el 30 por 100 en 1991- y, en general, por una diversificación de la dieta, que llega a cubrir satisfactoriamente el conjunto de las necesidades nutritivas de casi toda la población. En los años sesenta, según se desprende de la explotación de la encuesta de presupuestos familiares de 1964-1965, se cubren satisfactoriamente los requerimientos de energía, proteínas y de la mayor parte de micronutrientes, con un perfil calórico que se ajusta casi perfectamente a las recomendaciones, es decir, con una aportación energética de los hidratos de carbono del 53 por 100, de las proteínas del 12 por 100 y de los lípidos del 32 por $100^{53}$. El proceso, no obstante, no se detiene, y se observa en las últimas décadas una nueva problemática nutricional asociada al exceso y a los desequilibrios. Así se manifiesta, por una parte, en el desajuste del perfil calórico de la dieta española respecto al recomendado, en el cual en 1991 las grasas ya aportaban el 42 por 100 de la energía, la misma proporción que los hidratos de carbono. Por otra, en el excesivo peso de las proteínas de origen animal sobre el total, un 65 por 100 de los 93,5 gramos de proteína consumidos por persona y día en 1991, muy por encima del 50 por 100 recomendado sobre una ingesta también muy superior a las necesidades estimadas. Por último, se manifiesta en toda su gravedad en el aumento de la incidencia de la obesidad y de todas las enfermedades asociadas a los excesos y a los desequilibrios de la dieta.

\section{Bibliografía}

ABAD BALBOA, Carlos; GARCÍA DELGADO, José Luis, y MUÑOZ CIDAD, Cándido (1994): "La agricultura española en el último tercio del siglo XX: principales pautas evolutivas", en SUMPSI VIÑAS, José María (coord.), Modernización y cambio estructural en la agricultura española, Madrid, MAPA, pp. 69-125.

ALABERT, Francisco (1915): Encarecimiento de la vida en los principales países de Europa y singularmente en España, Memoria premiada con accésit por la Real Academia de Ciencias Morales y Políticas en el concurso ordinario de 1911, Madrid, Establecimiento Tipográfico de Jaime Ratés.

ALBURQUERQUE, Francisco (1981): "Métodos de control de la población civil: el sistema de racionamiento de alimentos y productos básicos impuesto en España tras la última guerra civil", en CASTILLO, Santiago, (ed.), Estudios sobre la historia de España. Homenaje a Tuñón de Lara, Tomo 2, Madrid, Ministerio de Educación y Ciencia, pp. 407-432. 
BALLESTEROS, Esmeralda (1997): Niveles de vida en España, siglos XIX y XX, tesis doctoral, Universidad Complutense de Madrid.

BEKAERT, Geert (1991): "Caloric Consumption in Industrializing Belgium", Journal of Economic History, 51, pp. 633-655.

CERDÀ, Ildefonso (1968-1971 [1867]): Teoría general de la urbanización, y aplicación de sus principios y doctrinas a la reforma y ensanche de Barcelona, vol. II, edición facsímil, Madrid, Instituto de Estudios Fiscales.

COLOMÉ, Josep (1996): L'evolució vitícola a la Catalunya del segle XIX. La comarca del Penedés, tesis doctoral, Universitat de Barcelona.

COLL, Sebastián, y QUIROGA, Gloria (1994): Height and the standard of living in 20th century in Spain: A preliminary report, Documentos de Trabajo del Departamento de Economía, Universidad de Cantabria.

COMISIÓN EXTRAPARLAMENTARIA PARA LA TRANSFORMACIÓN DEL IMPUESTO DE CONSUMOS (1910): Documentos y trabajos de la comisión consultiva para la transformación del impuesto de consumos, Tomo 1, Madrid, Minuesa de los Ríos.

CONARD, Pierre, y LOVETT, Albert (1969): “Problèmes de l'évaluation du coût de la vie en Espagne. I. Le prix du pain depuis le milieu du XIXe siècle", Mélanges de la Casa de Velázquez, 5, pp. 411-441.

CUSSÓ, Xavier (2001): Alimentació, mortalitat i desenvolupament. Evolució i disparitats regionals a Espanya des de 1860, tesis doctoral, Universitat Autònoma de Barcelona.

-(2005): "El estado nutritivo de la población española 1900-1970. Análisis de las necesidades y las disponibilidades de nutrientes", Historia Agraria, 36, pp. 329358.

CUSSÓ, Xavier, y GARRABOU, Ramon (2001): “Alimentació i nutrició al Vallès Oriental en les darreres dècades del segle XIX", Lauro, 21, pp. 26-34.

-(2003-04): “La transició nutricional a la Catalunya contemporània: una primera aproximació", Recerques, 47-48, pp. 51-80.

CUSSÓ, Xavier, y NICOLAU, Roser (2000): “La mortalidad antes de entrar en la vida activa en España. Comparaciones regionales e internacionales, 1860-1960", Revista de Historia Económica, 3, pp. 525-551.

DIRECCIÓN GENERAL DE ADUANAS (1896): Informe acerca de la producción, comercio y consumo del trigo en España redactado por la Dirección General de Aduanas y publicada por Real Orden de 20 de junio de 1896, Madrid, Sucesores de Rivadeneyra.

FALGUERAS, B. (1930): “La crisis del consumo de pan”, Panadería Catalana, Año II, 11, pp. 6-7.

FAOSTAT, $\underline{\text { http://faostat.fao.org/ }}$

FOGEL, Robert William (1993): “New Sources and New Techniques for the Study of Secular Trends in Nutritional Status, Health, Mortality, and the Process of Aging", Historical methods, vol. 26, 1, pp. 5-43. 
GARCÍA BARBANCHO, Alfonso (1960a): "Análisis de la alimentación española", Anales de economía, 66, pp. 72-119.

—(1960b): "Análisis de la alimentación española", Anales de economía, 67, pp. 271-363.

GARRABOU, Ramon, y PLANAS, Jordi (1998): Estudio agrícola del Vallés (1874), Granollers, Museu de Granollers.

Grupo de Estudios de Historia Rural (GEHR) (1991): Estadísticas históricas de la producción agraria española, 1859-1935, Madrid, MAPA.

GERMÁN, Luis (2006): “La evolución de la industria harinera en España durante el siglo XX", Investigaciones de Historia Económica, 4, pp. 139-176.

GIRAL PEREIRA, José (1914): Ración alimenticia desde los puntos de vista higiénico y social, Madrid, Librería Internacional, Adrián Romo Editor.

GÓMEZ MENDOZA, Antonio, y PÉREZ MOREDA, Vicente (1985): “Estatura y nivel de vida en la España del primer tercio del siglo XX", Moneda y Crédito, 174, pp. 29-64.

HERCBERG, Serge; ROUAUD, Catherine, y DUPIN, Henri (1988): “Evolución del consumo alimentario en Francia y en los países industrializados. Aspectos relativos a la Salud Pública", en HERCBERG, Serge, et al. (coord.), Nutrición y salud pública. Abordaje epidemiológico y políticas de prevención, Madrid, Ediciones CEA, pp. 27-48.

ISABEL SÁNCHEZ, José Luis (1994): “El rancho de cada día: una odisea del siglo XIX", Revista de Historia Militar, año XXXVIII, 77, pp. 107-154.

JUNTA CONSULTIVA AGRONÓMICA (1904): Estadística de la producción de cereales y leguminosas en el año 1903, Madrid.

-(1905): Estadística de la producción de cereales y leguminosas en el año 1904, Madrid.

-(1906): Estadística de la producción de cereales y leguminosas en el año 1905, Madrid.

LANA BERASAIN, José Miguel (2002): “Jornales, salarios, ingresos. Aproximación a la evolución de los niveles de vida desde la Navarra rural (1808-1935)", en MARTÍNEZ CARRIÓN, José Miguel (ed.), El nivel de vida en la España rural, siglos XVIII-XX, Alicante, Publicaciones de la Universidad de Alicante, pp. 183-233.

MAPA (1972-1998), Anuario de Estadística Agraria, Madrid.

MARTÍNEZ CARRIÓN, José Miguel (1991): “La estatura humana como un indicador del bienestar económico: un test local en la España del siglo XIX", Boletín de la $A D E H, 2$, pp. 51-78.

-(1994): "Niveles de vida y desarrollo económico en la España contemporánea: una visión antropométrica", Revista de Historia Económica, XII, 3, pp. 685-716.

MARTÍNEZ CARRIÓN, José Miguel, y PÉREZ CASTEJÓN, Juan José (1998): "Height and Standards of Living during the Industrialization of Spain: The Case of Elche", European Review of Economic History, 2, pp. 201-230.

MARTÍNEZ QUINTANILLA, Pedro (1865): La provincia de Gerona. Datos estadísticos, Girona, Imprenta de F. Dorca, sucesor de J. Grases. 
MOLINERO, Carme y YSÀS, Pere (1985): "Patria, justicia y pan". Nivell de vida i condicions de treball a Catalunya 1939-1951, Barcelona, Edicions la Magrana.

MOREAU DE JONNÉS, Alexandre (1835): Estadística de España, Barcelona, M. Rivadeneyra.

MORGADES, J. M. (comp. y ed.) (1935): Anuario Estadístico de Cereales 1935, Barcelona.

NICOLAU, Roser, y PUJOL, Josep (2005): “El consumo de proteínas animales en Barcelona entre las décadas de 1830 y 1930: evolución y factores condicionantes", Investigaciones de Historia Económica, 3, pp. 101-134.

QUIROGA VALLE, Gloria (2001): “Estatura, diferencias regionales y sociales y niveles de vida en España (1893-1954)", Revista de Historia Económica, XIX, núm. extraordinario, pp. 175-200.

SIMPSON, James (1989): "La producción agraria y el consumo español en el siglo XIX", Revista de Historia Económica, Año VII, pp. 355-388.

-(1997): La agricultura española 1765-1965: la larga siesta, Madrid, Alianza Editorial.

SMIL, Vaclav (2000): Feeding the World. A Challenge for the Twenty-First Century, Cambridge, Massachusetts, The MIT Press.

SOTILLA, Eduardo de la (1981 [1911]): "Producción y riqueza agrícola en España en el último decenio del siglo XIX y primero del XX", Agricultura y Sociedad, 18, pp. 331-409.

TOUTAIN, Jean Claude (1971): "La consommation alimentaire en France", Économies et sociétés. Cahiers de l'ISEA, Tome V, 11. pp. 1909-2049.

VARELA, Gregorio; MOREIRAS-VARELA, Olga, y VIDAL, Concepción (1968): "Niveles de nutrición en las diferentes regiones y estratos sociales", en VARELA, Gregorio (ed.), Contribución al estudio de la alimentación española, Granada, Instituto de Desarrollo Económico, pp. 17-64.

VARELA, Gregorio; GARCÍA RODRÍGUEZ, Domingo, y MOREIRAS-VARELA, Olga (1971): La nutrición de los españoles. Diagnóstico y recomendaciones, Madrid, Estudios del Instituto de Desarrollo Económico.

VARELA, Gregorio; MONTEAGUDO, Eva; CARVAJAL, Ángeles, y MOREIRAS, Olga (1991): El pan en la alimentación de los españoles, Eudema, Madrid.

VARELA, Gregorio (dir.) (1995): Encuesta de presupuestos familiares 1990-91. Estudio Nacional de Nutrición y Alimentación 1991, Madrid, INE.

VECCHI, Giovanni, y COPPOLA, Michela (2006): "Nutrition and Growth in Italy, 1861-1911. What Macroeconomic Data Hide", Explorations in Economic History, vol. 43, 3, pp 438-464.

YATES, P. Lamartine (1960): Food Production and Manpower in Western Europe, London, MacMillan. 